\title{
The Effects of Social Category Salience and Self-Construals upon Self-Stereotyping Strategies
}

\author{
KIRIKO SAKATA \\ Hiroshima University
}

\begin{abstract}
This study investigated the effect of gender salience, gender group membership, and independent/interdependent construals of self on self-defining strategies based on the research framework of Lorenzi-Cioldi (1991). Hypotheses were as follows. (1) self-stereotyping strategies tend to be employed under conditions of salient gender categories, while selfenhancement strategies defining self in positive terms will be mainly employed under conditions of salient individual differences. (2-a) Among self-stereotyping strategies, women, as dominated group-members, employ a gender-schema strategy processing male and female bipolarily, and (2-b) men, members of the dominant group, employ a self-schema strategy processing either male or female unipolarily. (3-a) Those with an interdependent construal of self, whose self is fundamentally connected with relevant others, employ a gender-schemata strategy that requires information on other groups, while (3-b) those with an independent construal of self, whose self is an autonomous entity independent of others, employ a selfschema strategy that does not require information on other groups. A test, measuring the response latencies of 79 subjects in self-descriptions on the BSRI (Bem, 1974) attributes was conducted, and showed support for hypotheses 2-a and 3-a, but since it evidenced no use of self-schema, hypotheses 2-b and 3-b were not supported. Since the effect of gender salience was not seen, hypothesis 1 was not supported. Considerations are offered as to experimental procedures and cultural differences.
\end{abstract}

Key words: self-stereotyping strategies, gender salience, independent / interdependent self-construals, gender-schema, self-schema, self-enhancement

Research on gender-identity has focused exclusively on stable personal factors considered as psychological traits. Most of the research has concerned gender identity as the psychological masculinity-femininity of individuals (Bem,
1974; Spence \& Helmreich, 1978), or the formation of cognitive schema categorizing people and phenomena through gender categories (Bem, 1981). Although such gender-identity considered as a trait has to some extent been cor-

The author is grateful to Professor Masaru Kurokawa of Hiroshima University for his helpful advice during the study.

Corresponce concerning this article should be addressed to Kiriko Sakata, Faculty of Integrated Arts and Sciences, Hiroshima University Higashi-Hiroshima, Hiroshima 739, Japan. 
roborated by occupational orientation or degree of success as a leader, many relevant findings have not been consistent.

It is clear that gender-identity considered as a psychological trait is a crucial variable in predicting social behavior. However, identity for membership in a gender group is not always salient, rather it becomes salient depending on the situations. Brown (1989) argued that all social behavior lies on a continuum extending from interpersonal settings to group settings. It is therefore possible to conceive that the identity of people moves along the continuum defined by the polarities of "as an individual" and "as a group". That is to say, if the intergroup situation is salient for men versus women, there is the possibility for self-stereotyping to occur regardless of masculinity-femininity. The inconsistency of findings in previous research about the relationships between gender-identity and social behavior seems to arise as a result of the divergent approaches to situational factors. It would therefore be significant to carry out more research on the influence of situational factors on gender identity.

Among the research carried out on genderidentity, the work of Lorenzi-Cioldi (1991) offers the most direct approach to the self-stereotyping process as related to gender. Lorenzi-Cioldi studied the effects of social and contextual factors on the self-stereotyping process from the perspective of social identity theory (Tajfel, 1982; Tajfel \& Turner, 1979). The social factor referred to was a gender categorization incorporating social position, namely, dominant male group and dominated female group. LorenziCioldi set a gender salient condition and an individual salient condition as contextual factors. In the former condition the gender category of women versus men was emphasized, while in the latter the individual differences were emphasized. It was predicted that self-stereotyping based on gender arises only in a gender salient condition, and that under an individual salient condition, a self-definition arises which is self- enhancing, in which the superiority of self is stressed in comparison with others.

Lorenzi-Cioldi considered that the genderstereotyping strategy of self differs in accordance with social position, and studied this using a response latency test for self-description. Two types of gender-stereotyping strategies were examined. The first is based on the definition of gender schema theory (Bem, 1984), which outlines the individual's readiness to encode and to organize information in terms of the male-female (in-group/out-group) dichotomous categorization. Using this male-female bipolar strategy, the subject's acceptant responses (hereinafter "me-response") to characteristics suitable for gender-stereotyping of one's own gender and rejective responses (hereinafter "notme-response") to characteristics fitting the opposite gender are fast, in comparison with other responses. The second strategy derives from self-schema theory (Markus, 1977; Markus, Crane, Bernstein \& Siladi, 1982). According to Markus, "one who develops a self-schema with respect to femininity becomes an expert in femininity, but there is little reason to assume that this person simultaneously becomes an expert in masculinity" (Markus, Crane, Bernstein \& Siladi, 1982, p.49). The self-defining strategy is therefore unipolar, and the me-response and notme-response toward characteristics of one's own gender are faster than those concerning characteristics of the opposite gender.

The social subordination of women elicits a need to efficiently learn and decode information about their superiors (Hall \& Halberstadt, 1981). By thus mastering the characteristics of female groups and male groups, women become susceptible to differentiating in-group and out-group, and so employ a bipolar gender-schema strategy. On the other hand, making up the dominant group, men have no need for information concerning the socially inferior group, and therefore stress the differentiation of self from other members of the in-group over differentiation between groups (Amancio, 1989; Lorenzi-Cioldi \& 
Doise, 1990). Men therefore employ a unipolar self-schema strategy. The results of LorenziCioldi's (1991) experiment supported the above hypotheses except the strategy of men employing self-schema.

Although the view proposed by LorenziCioldi (1991) is complex and based on numerous assumptions, the arguments that the gender identity salience varies contingently on the situational factors and that the asymmetry of men's and women's cognitive processes is derived from the difference of their social positions are of great importance. One purpose of this research is thus to establish that these apply as much to Japanese culture, traditionally thought of as very different from European and American culture, in respect of 'dominant men' and 'dominated women', as they do to these latter cultures.

Markus and Kitayama (1991) have proposed that cultures diverge in their systems for construing the self and others. According to them, within an independent, most typically Western construal, the self is constructed as an autonomous entity. Individuals will habitually seek the uniqueness of the self and, as a result, distinctive features of the self will be highly accessible. From an interdependent, most typically Asian or Eastern construal, the self is constructed as part of an encompassing network of social relationships. Individuals will habitually seek to organize their own thoughts, feelings, and behaviors in reference to the thoughts, feelings, and behaviors of others. As a result, distinctive features of the self will not be as accessible as those of others. Markus and Kitayama (1991) supported this analysis by their own experimental research, and they clarified the existence of individual differences of construal of self in identical cultural settings. The individual differences in such construals of self exist irrespective of gender group membership.

Even if the view of Lorenzi-Cioldi (1991) is generally applicable, the boundaries set for the categorization of women and men are unusually broad and thus do not account for individual differences within the categories. For example, it may be hypothesized that those with a construal of self as interdependent have a strong tendency to use gender-schema over self-schema, since people who display a consstrual of self as interdependent in their relations with others, which takes account of the mutual relationships between people (Markus \& Kitayama, 1991), will most probably pay attention to the characteristics and actions of others regardless of in-group/out-group membership. Conversely, those with a construal of self as independent tend to recognize themselves as an existence apart from others and situations, and thus have a tendency to employ unipolar self-schema, because when defining the self, they do not need to have information relative to others. The second purpose of this research is therefore to examine the impact of factors relating to individual differences, namely, construals of self as independent/interdependent, on the employment of strategies for self-definition.

This research makes 5 hypotheses: (1) With respect to the salient conditions for gender group membership, a self-stereotyping strategy is employed in relation to gender, and a self-enhancing strategy is used under the salient conditions for individual identity. (2-a) When self-stereotyping, women employ a strategy based on gender-schema. (2-b) When selfstereotyping, men employ a strategy based on self-schema. (3-a) When self-stereotyping, people with an interdependent construal of self employ strategies based on gender-schema. (3-b) When self-stereotyping, people with an independent construal of self, employ strategies based on self-schema.

\section{Method}

\section{Selections of the materials}

Sixty attributive words from the Bem Sex Role Inventory (BSRI: Bem, 1974) were used as stimuli in this study. Sixty five university students (39 males and 26 females) evaluated a de- 
gree of "social desirability" of each word according to a 5-point scale. As standard deviations of the resulting evaluation scores were rather large and thus inappropriate for the desirability criterion, scores evaluated by each of subjects participating in the experiment were used as the criterion for each subject. The another 40 undergraduate students (20 males and 20 females) classified the attributes into 3 categories (masculine, feminine, and sex-neutral) from the point of view of gender-stereotyping. Thus, 40 attributes (15 masculine, 15 feminine, and 10 sexneutral) were chosen and used as stimulus materials the experiment.

\section{Subjects and procedure}

The subjects were 40 male and 39 female undergraduate students aged 18 to 21 . Each subject was put into one of the following instructional sets. About half of the subjects were told they would be involved in a study "on similarities and differences between men and women" (gender-group salient conditon: GROUP), the other half would be involved in a study "on similarities and differences between individuals" (individual salient condition: IND). After receiving instructions, each subject was seated in front of a microcomputer. Then, she or he was asked to perform the following two tasks:

(a) Self-description assignment: The subjects judged whether 40 attributes showed at random one by one on the display applied to them or not. In case an attribute was self-descriptive ("applies to me"), the subject responded by pressing the key labelled 1 . In case it was otherwise ("does not apply to me"), she/he responded by pressing the key labelled 2. Though response latencies were measured at the same time, the subjects were not made aware of this fact.

(b) Social desirability evaluation assignment: As with the self-description assignment, subjects evaluated each attribute sequentially shown on the display as to the degree of social desirability of the word he/she judged by pressing one key out of 5 keys labelled from 1 ('not desirable at all') to 5 ('very desirable').

All subjects were assigned Task (a) before Task (b). After the tasks were completed, each subject was asked to fill in the Hiroshima University version of the self-construal scale (Kurokawa, 1994), consisting of 6 independency and 6 interdependency items, developed with reference to those given by Markus \& Kitayama (1991).

\section{Criteria of using self-defining strategies}

In case response latencies which conform to related strategies (consistent information) are smaller than those which do not conform (inconsistent information), the strategies were used. In gender-schema strategies, consistent information would be "me-response to attributes of one's own sex and not-me-response to those of the opposite sex." Inconsistent information would be "me-response to attributes of the opposite sex and not-me-response to those of one's own sex." In self-schema strategies, consistent information would be "me- and not-me-responses to attributes of one's own sex" and inconsistent information would be "me- and not-me-responses to those of the opposite sex." In self-enhancement strategies, consistent information would be "meresponse to socially desirable attributes and notme-response to socially undesirable attributes" and inconsistent information would be "not-meresponse to socially desirable attributes and meresponse to socially undesirable attributes."

\section{Results}

\section{Independent-interdependent construals of self}

The mean values of the scores for independence and interdependence of the subjects were, respectively, $19.3(S D=3.52)$ and $21.6(S D=$ 3.14). For each of the subjects a differentiation score for "independence minus interdependence" was calculated, and used in an analysis including construals of self with a point distribution of the top $40 \%$ (4 points or more) for interdependent subjects and the bottom $40 \%$ (1 point or less) for independent subjects. It may be noted 
The Effects of Social Category Salience and Self-Construals upon Self Stereotyping Strategies

Table 1

Means of "me" response rate to gender-related attributes for each group

\begin{tabular}{lccccc}
\hline & \multicolumn{2}{c}{ Male } & & \multicolumn{2}{c}{ Female } \\
\cline { 2 - 3 } \cline { 5 - 5 } Attributes & Independent & Interdependent & & Independent & Interdependent \\
\hline \multirow{3}{*}{ Masculine } & 56.0 & Gender group salient & & \\
Feminine & 51.3 & 25.8 & & 31.3 & 12.2 \\
Neutral & 59.0 & 54.2 & & 54.7 & 65.6 \\
& & 65.0 & & 53.0 & 61.7 \\
Masculine & 43.3 & Individual salient & & \\
Feminine & 57.8 & 42.2 & & 22.2 & 16.7 \\
Neutral & 56.6 & 67.4 & 61.1 & 65.8 \\
\hline
\end{tabular}

Note. The values represent mean percentages of "me" response to given attributes.

here that no difference was observed with respect to the ratio of women vs. men included in interdependent or independent subjects.

\section{Content of self-definition}

To test whether or not there occurred any differentiation in the content of self-definition in the subjects under GROUP setting and IND setting, the me-response rate of each subject toward each attribute was calculated. The mean values for each condition are shown in Table 1. Considering the tendency of the subjects as a whole, with respect to gender-stereotyping, meresponse to neutral attributes constitute a majority (62\%), followed by feminine attributes (57\%) and masculine attributes (32\%). Regarding the points for socially desirable attributes, the me-response to positive attributes constitute a majority (55\%), followed, in order, by neutral attributes (44\%) and negative attributes (40\%). (Concerning socially desirable attributes, the subjects rated themselves 4 or 5 for positive attributes, 3 for neutral attributes, and 1 or 2 for negative attributes.) With me-response rates to masculine, feminine, and neutral attributes as dependent variables, a 3-way analysis of variance was conducted of employing salient conditions (GROUP/IND) $\times$ gender (man/women) $\times$ self construals (independent/interdependent).
For masculine attributes, the main effect of gen$\operatorname{der}[F(1,55)=14.734, p<.001]$, the main effect of self construals $[F(1,55)=6.391, p<.05]$, and the marginal interaction of salient condition $\times$ self construals $[F(1,55)=3.710, p<.10]$ were significant. The me-response rate toward masculine attributes was higher for men $(M=$ 42.1\%) than for women ( $M=19.4 \%)$, and higher for independent subjects $(M=39.6 \%)$ than interdependent subjects $(M=25.6 \%)$. Additionally, according to the test of simple main effects, while for GROUP setting, independent subjects ( $M=43.7 \%)$ were higher than interdependent subjects $(M=20.0 \%)$, no difference was observed between the two groups with respect to IND setting (see Figure 1). From the above results, it can be stated that although as a whole men and women both tend to describe the self in terms of gender neutral attributes, men show a higher acceptance rate of masculine attributes than women. On the other hand, no difference was observed for acceptance rates concerning feminine and neutral attributes. Further, there was a much higher acceptance of masculine attributes by independent subjects as compared with interdependent subjects, and this tendency was significant under GROUP setting. An explanation of this interaction will be discussed later. 


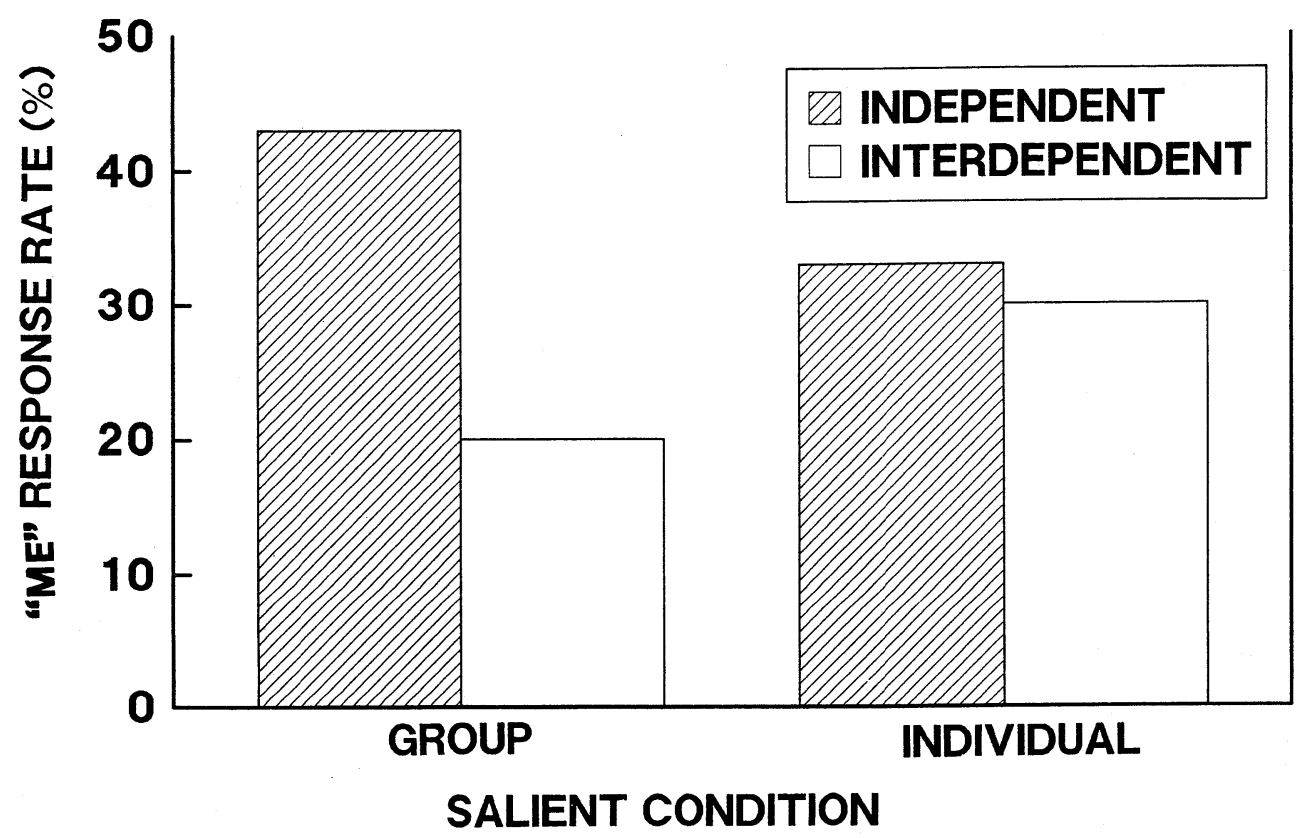

Figure 1. Means of "me" response rate to masculine attributes.

Similar analysis were conducted regarding me-response to positive, negative and neutral social desirability. However, no significant results were obtained.

\section{Self-defining strategies}

Gender-schema strategy. In order to support hypotheses 2-a and 3-a, the reaction time of consistent information should be significantly smaller than that of inconsistent information for women and interdependent subjects, and there should be no significant differences in the reaction time for either men or independent subjects. Furthermore, with such an interaction only seen under GROUP setting, hypothesis 1 can also be supported. A gender $\times$ information consistency (consistent/inconsistent) ANOVA was done with logarithmically transformed reaction time as the dependent variable, and the interaction was significant $[F(1,77)=$ 21.231, $p<.001$ ] (see Figure 2). Among females, the simple main effect of consistency of information was significant $[p<.001]$. Employing the gender-schema strategy, women exhibited a lower reaction time for consistent information than for inconsistent information. This result supports hypothesis 2-a. Furthermore, conducting a 2 (gender) $\times 2$ (self construals) $\times 2$ (information consistency) ANOVA, the three way interaction was marginally significant $[F(1,59)=3.110, p<.10]$ (see Figure 3 ). The results of the tests of simple interactions and the tests of simple simple main effects were as follows: Though women used gender-schema strategies regardless of self construals, interdependent women especially showed a greater differentiation of consistent and inconsistent information than independent women, clearly ex- 


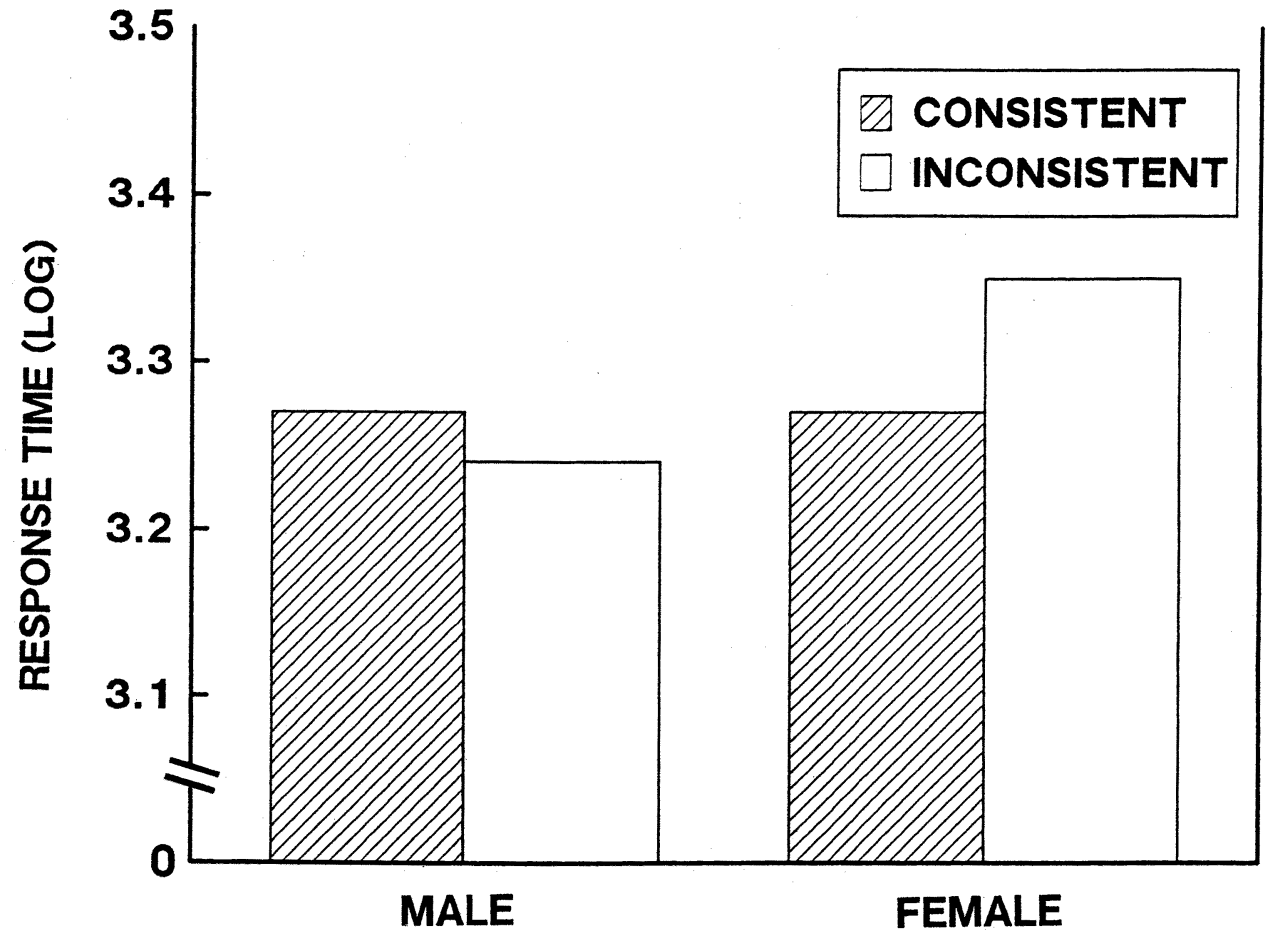

Figure 2. Means of logarithmic transformed response time by male and female in the framework of gender-schema strategy.

hibiting the use of gender-schema strategy. The use of gender-schema strategy by independent men was not seen, but interdependent men did display a reverse gender-schema (i.e., the same gender-shcema as women) in reacting quickly to inconsistent information. In any case, support for hypothesis 3-a was found by the greater use of gender-schema required for out-group information by interdependent subjects than by independent subjects. An analysis including salient condition was conducted, but since no significant results were seen, it did not support hypothesis 1.

Self-schema strategy. For men and independent subjects, the reaction time for consistent information should be significantly less than for inconsistent information, and as for both women and interdependent subjects, there should be no significant diffrentiation in reaction time in order to support hypotheses 2-b and 3-b. Further, such interaction as observed only under GROUP setting would support hypothesis 1 . A 2 (gender) $\times 2$ (information consistency) ANOVA was carried out with logarithmically transformed reaction time as a dependent variable, and the interaction was significant $[F(1,77)=4.500, p<$ $.05]$. However, the test of simple main effects showed that this interraction was caused by the quick reaction time for women responding to inconsistent information, and did not show the use of self-schema strategy by men. Accordingly, hypothesis 2-b was not supported. Significant results were not observed for the salient condition $\times$ gender $\times$ information consistency, and gender $\times$ self construals $\times$ information consistency ANOVA. Because the influence of salient 


\section{Q CONSISTENT $\square$ INCONSISTENT}

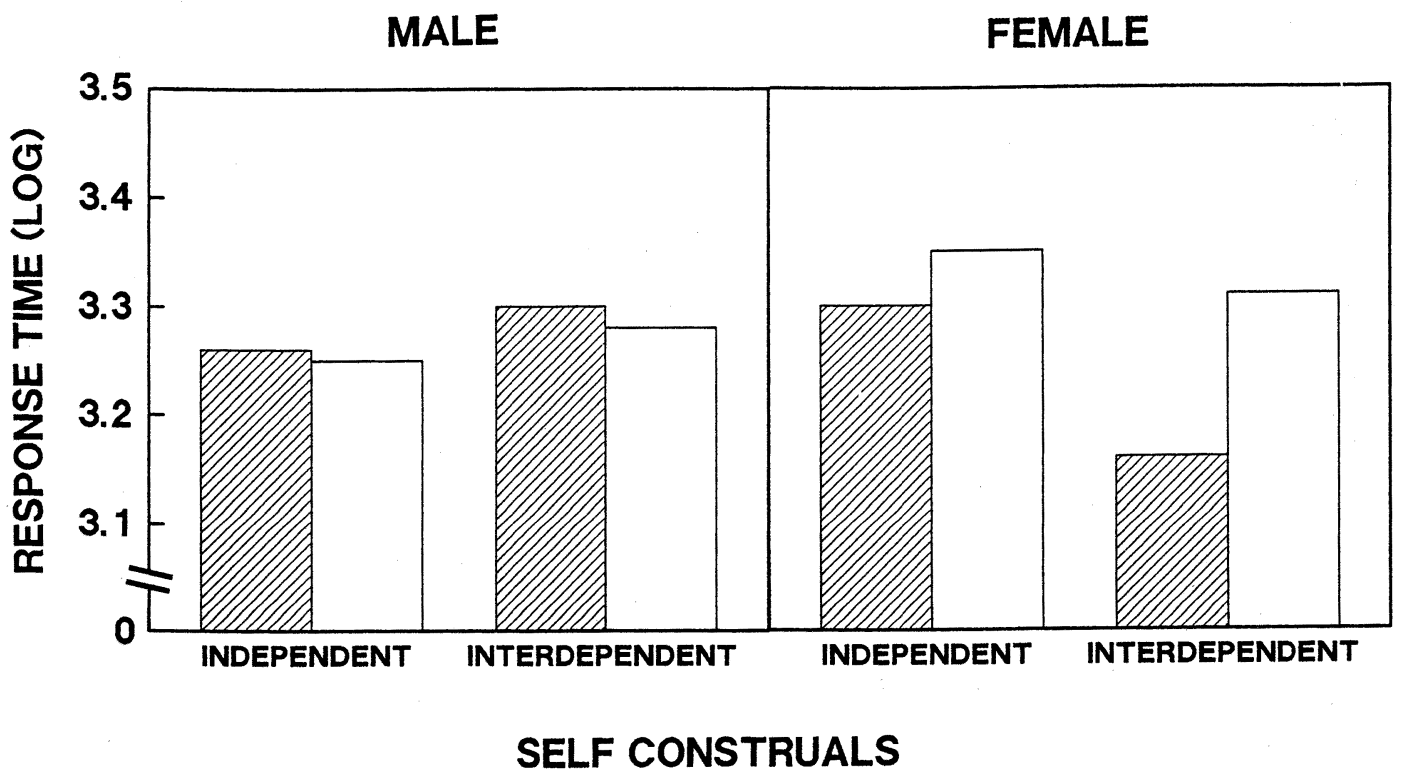

Figure 3. Means of logarithmic transformed response time by each self-construal group in the framework of gender-schema strategy.

condition and self construals were not observed, hypotheses 1 and 3-b were not supported.

Self-enhancement strategy. In support of hypothesis 1 , under IND setting, those with reaction times for consistent information should be less than for inconsistent information, and that tendency should be greater than for GROUP setting. A 2 (salient condition) $\times 2$ (gender) $\times$ 2 (information consistency) ANOVA was carried out with logarithmic transformed reaction time as a dependent variable, and the interaction for gender $\times$ information consistency was marginally significant $[F(1,75)=3.390, p<.10]$ (see Figure 4). Only the simple main effect of information consistency on male was significant $[p<.05]$. While men used self-enhancement strategies, women did not. This result had not been expected. However, the effects of a salient condition were not observed. As a consequence, hypothesis 1 was not supported.

\section{Reexamination of the effects of self construals.}

In the above analysis, the effects of self construals on content of self-definition (i.e., meresponse rates to masculine attributes: see Figure 1) and use of gender-schema strategy (see Figure 3) were observed. However, in this experiment, among the words denoting masculine attributes there are those such as "independent", which connote independency, and some words which implied interdependency (e.g., "sympathetic") were included among feminine attributes. The fact suggests that the masculine-feminine dimension of the stimulative 


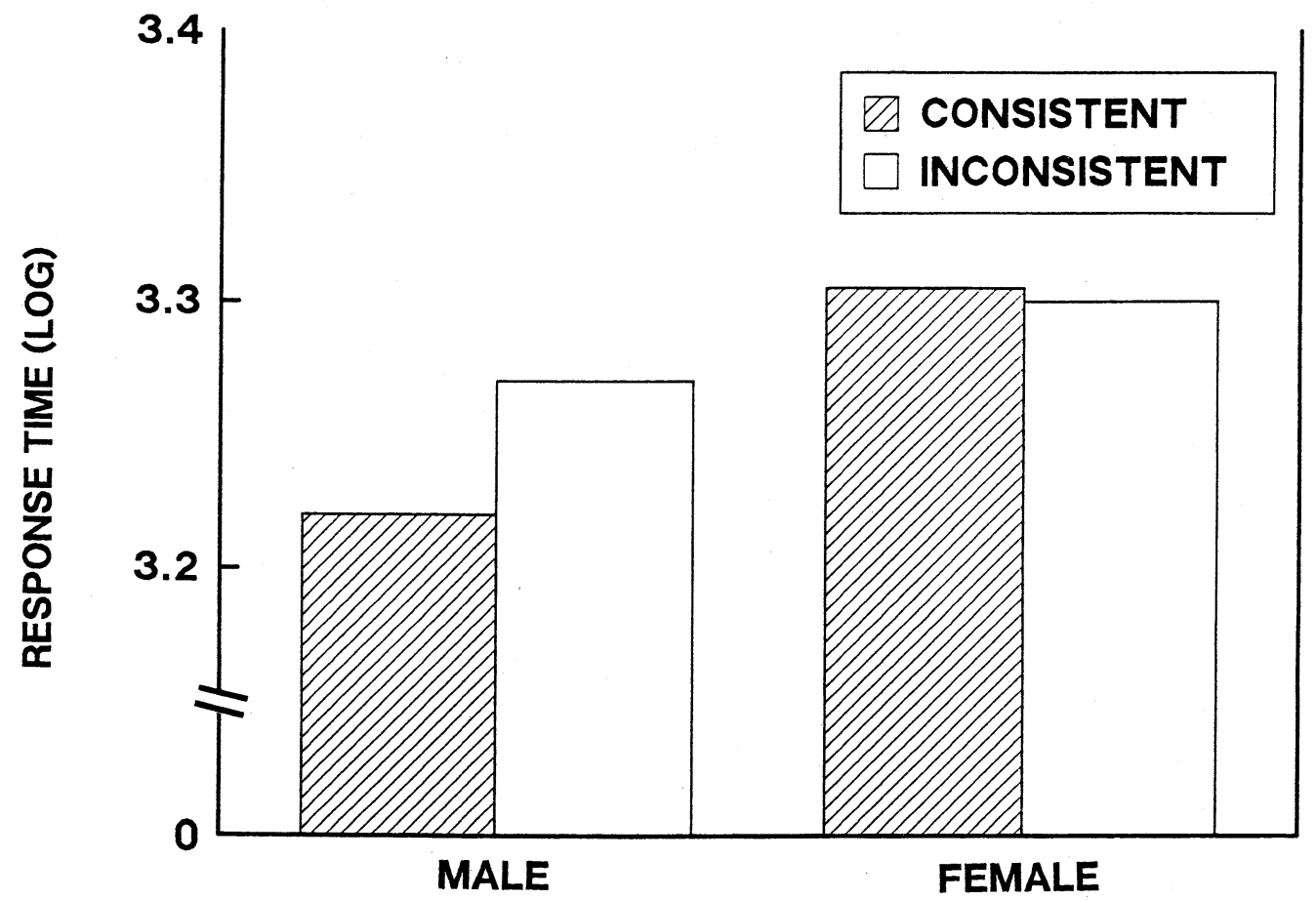

Figure 4. Means of logarithmic transformed response time in the framework of self-enhancement strategy.

words which were used and the independentinterdependent dimension have not been clearly distinguished. Considering the possibility that independent or interdependent subjects possessed schema related to the independentinterdependent dimension, the effects of self construals should be due to the independent or interdependent connotations contained in gender related attributes. Indeed, interdependent subjects used bipolar gender-schema strategies more cleary than independent subjects, but interdependent men used a reverse gender-schema (i.e., the same gender-schema as women) in reacting quickly to inconsistent information. This raises the possibility that the interaction effect was caused by rapid responses on the part of these male subjects to "interdependent attributes", but not to "feminine attributes". Fur- ther, the interaction of a salient condition and self construals, difficult to understand, may be caused by the fact that under conditions which emphasize gender, independent subjects identify themselves with "independent" male groups, but not with "interdependent" female groups.

To avoid the above confusion, I reexamined the effect of self construals on me-response rates to masculine attributes and use of genderschema excluding the three words "independent", "individualistic", and "self-reliant" from the masculine attributes, and the three phrases "eager to soothe hurt feelings", "sensitive to the needs of others", and "sympathetic" from feminine attributes. Concerning the me-response rate to masculine attributes, as a result, the interaction of salient condition $\times$ self construals $[F(1,55)=5.575, p<.05]$ turned out to be sig- 
nificant. On the other hand, the 3 way interaction (i.e., gender $\times$ self construals $\times$ information consistency) on the use of gender-schema strategy was not significant $[F(1,59)=1.660$, $p=.20]$. Thus, there seems to be a possibility that a part of the effect of self-construals on the use of gender-schema strategy was due to independent-interdependent connotation contained in the stimulus words.

\section{Discussion}

In this research, an unexpected interaction was observed between salience conditions and self construals only in the content of self-description; however, there was no evidence of the effect of salient conditions on self-defining strategies. This result differs from that of Lorenzi-Cioldi (1991). The reason may be the weak impact of the manipulation of salience conditions. Lorenzi-Cioldi (1991) provided 3 tasks to the subjects; (a) selfdescription, (b) social desirability evaluations, and (c) gender-stereotyping judgments classified as masculine, feminine, and sex-neutral. Under the conditions of gender group, the tasks were carried out in the order of (c), (a), (b), and under the conditions of individual differences in the order of (a), (c), (b), so as to reinforce the impact of the instructions. Because the present study omitted task (c), the manipulation of salient conditions could be done only through the instructions, and the resulting impact was consequently weakened. If the same procedure as in Lorenzi-Cioldi (1991) were to be used, the effect of gender salient conditions would be observed. The explanation of the interaction that was observed regarding the content of self-description is complex. After the three 'independent' words from masculine stimulus words were excluded, regardless of gender, independent people gave many me-responses to masculine attributes under gender group conditions. If attributes connoting independence are intrinsically contained in the concept of masculinity, it seems to be difficult to separate the one from the other. Thus, the method of excluding three 'independent' words might be insufficient to separate the one from the other in regard to those concepts. It may therefore be thought that under conditions which emphasise gender, independent subjects identify themselves with male or masculine groups.

As for the hypothesis relating to self-defining strategies and gender as a variable of social position, there was support for the proposition that women use gender-schema strategies, but there was no support for the proposition that men use self-schema strategies. These results are consistent with the findings of Lorenzi-Cioldi (1991). The tendency for women to employ a bipolar gender-schema strategy would appear to be universal across cultures. Moreover, it suggests that the hypothesis on the use of selfschema strategies by men needs to be reconsidered. Lorenzi-Cioldi (1991) raised two possibilities as to why the use of self-schema was not proven. The first was that in the instructions for gender group conditions, the male vs. female dichotomy was stressed more than necessary. The second possibility was that the men were not as sensitive to gender-related intergroup boundaries as were the women. The former possibility would not be applicable in the case of the present research, since it is considered that the impact of the instructions themselves was weakened. However, with regard to the latter possibility, this research could be considered relevant. The difference in sensitivity to intergroup boundaries according to gender can be explained with reference to where men and women stand in the social hierarchy. In other words, because men, who constitute the dominant group, favor interpersonal differentiation as well as intergroup differentiation, they have no need of being particularly sensitive to intergroup boundaries. There is also a third possibility, and that is the problem of the appropriateness of self-schema as a self-stereotyping strategy in relation to gender-related categories. Unlike the categories of race or nationhood, 
the category of gender consists of only two discrete levels. Thus it seems to be difficult for the unipolar information processing strategy to be carried out.

With regard to the hypothesis concerning construals of self as a personal factor, there was also support regarding gender-schema strategy. While this showed the validity of the view of Hall \& Halberstadt (1981), it also provided a suggestion concerning cultural differences. That is to say, people from Eastern cultural spheres characterized by interdependency, generally seem to use bipolar gender-schema strategies more than those from Western cultures, which tend to emphasize independence. However, reexamination suggested that a part of the effect of self-construals on the use of genderschema strategies was caused by a covariation in the independency-interdependency parameters and the masculinity-femininity parameters. Though it may be difficult to separate these parameters completely, the hypothesis concerning construals of self should be examined further by taking more effective treatment to clearly distinguish them.

Concerning self-enhancement strategies, the observation that men and women give asymmetrical results is of particular interest. As for the results of Lorenzi-Cioldi (1991), there was no evidence of gender differences in the use of selfenhancement. Concerning the absence of use of self-enhancement by women, it will be necessary to conduct further studies taking into account previous findings such as Takata's (1987) "selfdeprecative tendencies of Japanese people".

\section{References}

Amancio, L. (1989). Social differentiation between 'dominant' and 'dominated' groups: Toward an integration of social stereotypes and social identity. European Journal of Social Psychology, 19, 1-10.

Bem, S. L. (1974). The measurement of psychological androgyny. Journal of Consulting and Clinical Psychology, 42, 155-162.
Bem, S. L. (1981). Gender schema theory: A cognitive account of sex typing. Psychological Review, 88, 354-364.

Brown, R. J. (1988). Group processes: Dynamics within and between groups. Oxford: Basil Blackwell.

Hall, J. A., \& Halberstadt, A. G. (1981). Sex roles and nonverbal communication skills. Sex Roles, 7, 273-287.

Kurokawa, M. (1994). The effects of the interdependency and the construals of self upon one's interpersonal influential behaviors. Report of Research Project, Grant-in Aid for Scientific Research (C), 17-20.

Lorenzi-Cioldi, F., \& Doise, W. (1990). Levels of analysis and social identity. In D. Abram \& M. Hogg (Eds.), Social identity theory: constructive and critical advances. Harvester Press, London.

Lorenzi-Cioldi, F. (1991). Self-stereotyping and self-enhancement in gender groups. European Journal of Social Psychology, 21, 403417.

Markus, H. (1977). Self-schemata and processing information about the self. Journal of Personality and Social Psychology, 35, 6378.

Markus, H., Crane, M., Bernstein, S., \& Siladi, M. (1982). Self-schemas and gender. Journal of Personality and Social Psychology, 42, 38-50.

Markus, H., \& Kitayama, S. (1991). Culture and the self: Implications for cognition, emotion, and motivation. Psychological Review, 98, 224-253.

Spence, J. L., \& Helmreich, R. L. (1978). Masculinity and femininity: Their psychological dimensions, correlates and antecedents. Austin: University of Texas Press.

Tajfel, H., \& Turner, J. C. (1979). An integrative theory of intergroup conflict. In W. Austin \& S. Worchel (Eds.), The Social Psychology of Intergroup Relations, Brooks/Cole, 33-47.

Tajfel, H. (1982). Social psychology of inter- 


\section{Kiriko Sakata}

group relations. Annual Review of Psychology, 33, 1-30.

Takata, T. (1987). Self-deprecative tendencies in self evaluation through social compari- son. The Japanese Journal of Experimental Social Psychology, 27, 27-36.

(Received Dec. 19, 1994; Accepted Jan. 30, 1995) 